Supporting Information

\title{
Comparison of bioavailability and biotransformation of inorganic and organic arsenic to two marine fish
}

\author{
Wei Zhang, Wen-Xiong Wang, Li Zhang*
}

Pages S1-S5

\section{List of Figures}

Figure S1 The AsB distribution (\%) in exposed diets and different tissues of rabbitfish and seabass after different exposed treatments for $28 \mathrm{~d}$....

Figure S2 The correlation between newly accumulated As concentrations and the ratio of organic As (subtracting the background concentrations)/inorganic As in seabass after $28 \mathrm{~d}$ exposure.

\section{List of Tables}

Table S1 Total As, As species concentrations and distribution (\%) in unspiked food. Values are means $\pm \mathrm{SD}(\mathrm{n}=3)$. The foods consist of artificial diets, red algae $G$. lemaneiformis, G.gigas, green algae $U$. lactuca, polychaete $N$. succinea, oyster $S$. cucullata, and clam A. violascens. As(III), arsenite; As(V), arsenate; MMA, monomethylarsonate; DMA, dimethylarsinate; AsB, arsenobetaine. BDL (below detection limit) S3

Table S2 As species concentrations in intestine, liver, and muscle tissues of marine rabbitfish after different dietborne exposure for $28 \mathrm{~d}$. Values are means $\pm \mathrm{SD}(\mathrm{n}=3)$. The foods consist of artificial diets, G. lemaneiformis, G.gigas, and U. lactuca......S4

Table S3 As species concentrations in intestine, liver, and muscle tissues of marine seabass after different dietborne exposure for $28 \mathrm{~d}$. Values are means $\pm \mathrm{SD}(\mathrm{n}=3)$. The foods consist of artificial diets, polychaete, oyster, and clam......................S5 


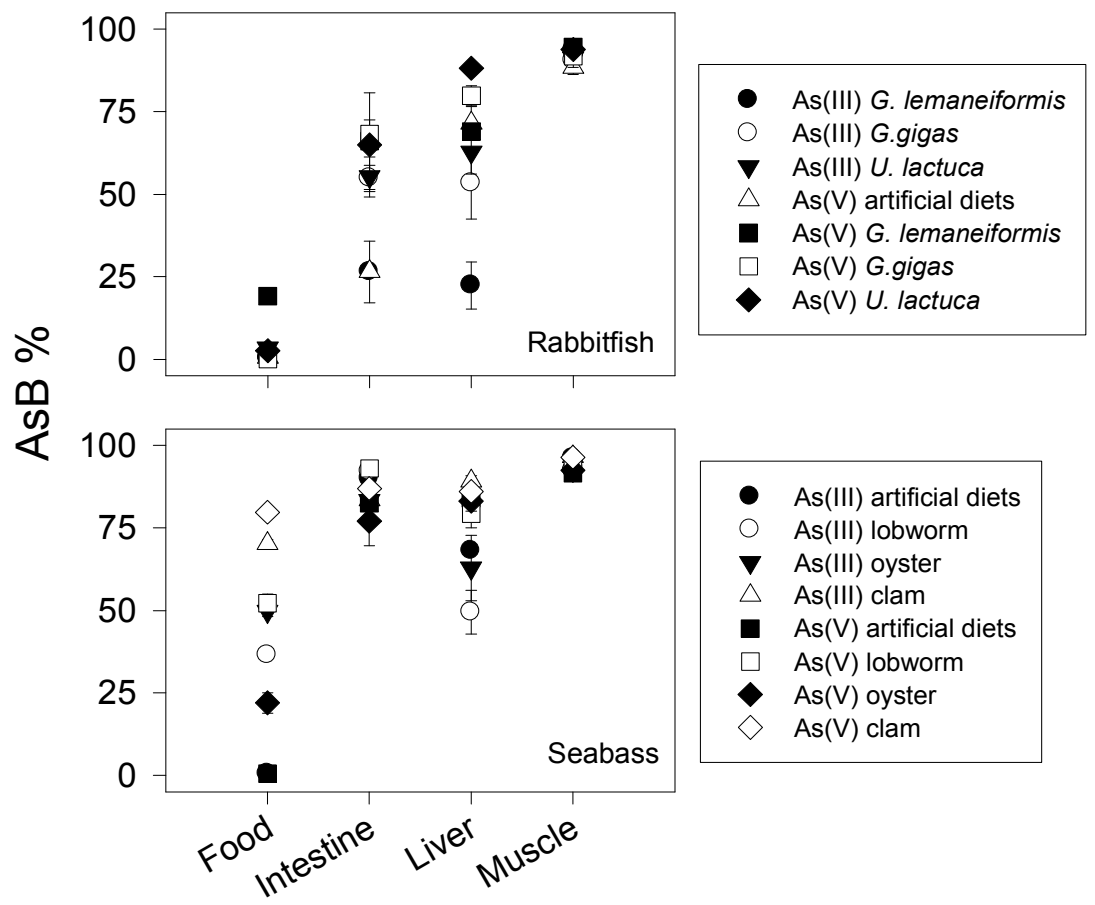

Figure S1 The AsB distribution (\%) in exposed diets and different tissues of rabbitfish and seabass after different exposed treatments for $28 \mathrm{~d}$ 


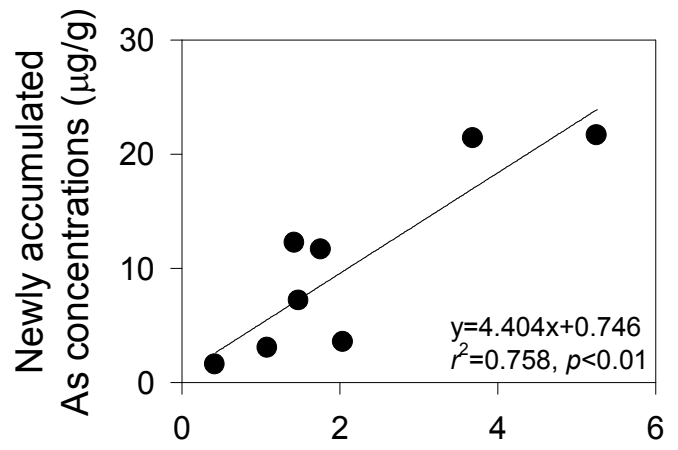

The ratio of organic As/inorganic As in fish

Figure S2 The correlation between newly accumulated As concentrations and the ratio of organic As (subtracting the background concentrations)/inorganic As in seabass after $28 \mathrm{~d}$ exposure 
Table S1 Total As, As species concentrations and distribution (\%) in unspiked food. Values are means $\pm \mathrm{SD}(\mathrm{n}=3)$. The foods consist of artificial diets, red algae $G$. lemaneiformis, G.gigas, green algae $U$. lactuca, polychaete $N$. succinea, oyster $S$. cucullata, and clam A. violascens. As(III), arsenite; As(V), arsenate; MMA, monomethylarsonate; DMA, dimethylarsinate; AsB, arsenobetaine. BDL (below detection limit).

\begin{tabular}{|c|c|c|c|c|c|c|c|}
\hline \multicolumn{7}{|c|}{ As species concentrations $(\mu \mathrm{g} / \mathrm{g})$ and distribution $(\%)$} & \multirow{2}{*}{ Total As } \\
\hline & $\mathrm{As}(\mathrm{III})$ & $\operatorname{As}(\mathrm{V})$ & MMA & DMA & AsB & Unidentified As & \\
\hline \multirow{2}{*}{ Artificial diets } & $0.11 \pm 0.03$ & $0.18 \pm 0.03$ & $0.01 \pm 0.00$ & $0.03 \pm 0.01$ & $0.26 \pm 0.06$ & & \multirow{2}{*}{$0.57 \pm 0.11$} \\
\hline & $(19.0 \pm 0.61 \%)$ & $(30.5 \pm 0.70 \%)$ & $(2.39 \pm 1.09 \%)$ & $(5.00 \pm 0.64 \%)$ & $(43.1 \pm 0.54 \%)$ & BDL & \\
\hline \multirow[t]{2}{*}{ G. lemaneiformis } & $0.01 \pm 0.03$ & $0.61 \pm 0.04$ & & $0.81 \pm 0.06$ & & $2.16 \pm 0.56$ & \multirow{2}{*}{$3.88 \pm 0.16$} \\
\hline & $(0.30 \pm 0.03 \%)$ & $(16.9 \pm 0.47 \%)$ & BDL & $(22.6 \pm 1.24 \%)$ & BDL & $(60.2 \pm 2.13 \%)$ & \\
\hline \multirow[t]{2}{*}{ G. gigas } & $0.01 \pm 0.00$ & $1.29 \pm 0.03$ & & $1.36 \pm 0.45$ & & $3.56 \pm 0.34$ & \multirow{2}{*}{$6.41 \pm 1.48$} \\
\hline & $(0.21 \pm 0.02 \%)$ & $(20.1 \pm 0.37 \%)$ & BDL & $(21.2 \pm 0.98 \%)$ & BDL & $(58.4 \pm 1.36 \%)$ & \\
\hline \multirow[t]{2}{*}{ U. lactuca } & $0.01 \pm 0.00$ & $1.20 \pm 0.06$ & & $0.55 \pm 0.09$ & & $1.24 \pm 0.30$ & \multirow{2}{*}{$3.17 \pm 0.15$} \\
\hline & $(0.40 \pm 0.02 \%)$ & $(40.0 \pm 0.67 \%)$ & BDL & $(18.2 \pm 1.21 \%)$ & BDL & $(41.4 \pm 0.34 \%)$ & \\
\hline \multirow[t]{2}{*}{ Polychaete } & $0.09 \pm 0.01$ & $0.07 \pm 0.02$ & $0.08 \pm 0.00$ & & $7.03 \pm 0.90$ & & \multirow{2}{*}{$6.98 \pm 0.92$} \\
\hline & $(1.18 \pm 0.21 \%)$ & $(0.93 \pm 0.145 \%)$ & $(1.08 \pm 0.15 \%)$ & BDL & $(94.9 \pm 2.91 \%)$ & $\mathrm{BDL}$ & \\
\hline \multirow[t]{2}{*}{ Oyster } & & $0.05 \pm 0.01$ & & $0.55 \pm 0.07$ & $14.8 \pm 0.23$ & & \multirow{2}{*}{$16.2 \pm 1.24$} \\
\hline & BDL & $(0.31 \pm 0.03 \%)$ & BDL & $(3.40 \pm 0.34 \%)$ & $(91.5 \pm 1.53 \%)$ & BDL & \\
\hline \multirow[t]{2}{*}{ Clam } & & $0.04 \pm 0.01$ & & $0.44 \pm 0.11$ & $9.58 \pm 0.97$ & & \multirow{2}{*}{$10.3 \pm 1.21$} \\
\hline & $\mathrm{BDL}$ & $(0.39 \pm 0.02 \%)$ & BDL & $(4.27 \pm 0.46 \%)$ & $(93.0 \pm 2.13 \%)$ & $\mathrm{BDL}$ & \\
\hline
\end{tabular}


Table S2 As species concentrations in intestine, liver, and muscle tissues of marine rabbitfish after different dietborne exposure for $28 \mathrm{~d}$. Values are means $\pm \mathrm{SD}(\mathrm{n}=3)$. The foods consist of artificial diets, G. lemaneiformis, G.gigas, and U. lactuca.

\begin{tabular}{lccccc}
\hline & \multicolumn{5}{c}{ As species concentrations $(\mu \mathrm{g} / \mathrm{g})$} \\
\hline & $\mathrm{As}(\mathrm{III})$ & $\mathrm{As}(\mathrm{V})$ & $\mathrm{MMA}$ & $\mathrm{DMA}$ & $\mathrm{AsB}$ \\
\hline & & Intestine & & \\
Control & $0.03 \pm 0.02$ & $0.05 \pm 0.01$ & $0.01 \pm 0.00$ & $0.02 \pm 0.00$ & $0.55 \pm 0.02$ \\
As(III) G. lemaneiformis & $0.29 \pm 0.08$ & $0.34 \pm 0.11$ & $0.04 \pm 0.03$ & $0.26 \pm 0.09$ & $0.33 \pm 0.11$ \\
As(III) G.gigas & $0.11 \pm 0.03$ & $0.18 \pm 0.01$ & $0.03 \pm 0.01$ & $0.14 \pm 0.06$ & $0.54 \pm 0.02$ \\
As(III) U. lactuca & $0.13 \pm 0.05$ & $0.13 \pm 0.05$ & $0.03 \pm 0.00$ & $0.31 \pm 0.03$ & $0.74 \pm 0.09$ \\
As(V) artificial diets & $0.10 \pm 0.01$ & $0.37 \pm 0.05$ & $0.03 \pm 0.00$ & $0.10 \pm 0.05$ & $0.21 \pm 0.00$ \\
As(V) G. lemaneiformis & $0.05 \pm 0.02$ & $0.18 \pm 0.01$ & $0.03 \pm 0.00$ & $0.21 \pm 0.15$ & $0.93 \pm 0.28$ \\
As(V) G.gigas & $0.04 \pm 0.02$ & $0.21 \pm 0.01$ & $0.03 \pm 0.01$ & $0.19 \pm 0.12$ & $0.99 \pm 0.01$ \\
As(V) U. lactuca & $0.08 \pm 0.02$ & $0.20 \pm 0.01$ & $0.02 \pm 0.00$ & $0.08 \pm 0.00$ & $0.69 \pm 0.02$ \\
& & Liver & & & \\
Control & $0.29 \pm 0.03$ & $0.23 \pm 0.03$ & BDL & $0.12 \pm 0.01$ & $0.50 \pm 0.13$ \\
As(III) G. lemaneiformis & $0.53 \pm 0.14$ & $0.40 \pm 0.07$ & $0.04 \pm 0.01$ & $0.26 \pm 0.06$ & $0.35 \pm 0.06$ \\
As(III) G.gigas & $0.26 \pm 0.05$ & $0.20 \pm 0.05$ & BDL & $0.13 \pm 0.04$ & $0.69 \pm 0.25$ \\
As(III) U. lactuca & $0.37 \pm 0.20$ & $0.21 \pm 0.02$ & BDL & $0.07 \pm 0.03$ & $1.08 \pm 0.02$ \\
As(V) artificial diets & $0.22 \pm 0.05$ & $0.15 \pm 0.00$ & BDL & $0.07 \pm 0.03$ & $1.09 \pm 0.05$ \\
As(V) G. lemaneiformis & $0.23 \pm 0.04$ & $0.21 \pm 0.06$ & BDL & $0.07 \pm 0.01$ & $1.13 \pm 0.18$ \\
As(V) G.gigas & $0.10 \pm 0.02$ & $0.12 \pm 0.07$ & BDL & $0.04 \pm 0.02$ & $1.05 \pm 0.08$ \\
As(V) U. lactuca & $0.10 \pm 0.02$ & $0.11 \pm 0.01$ & BDL & $0.04 \pm 0.00$ & $1.77 \pm 0.15$ \\
& & Muscle & & & \\
Control & $0.05 \pm 0.01$ & $0.04 \pm 0.02$ & $0.01 \pm 0.00$ & $0.02 \pm 0.01$ & $1.49 \pm 0.14$ \\
As(III) G. lemaneiformis & $0.06 \pm 0.02$ & $0.05 \pm 0.03$ & $0.01 \pm 0.01$ & $0.01 \pm 0.01$ & $1.97 \pm 0.00$ \\
As(III) G.gigas & $0.08 \pm 0.03$ & $0.05 \pm 0.02$ & $0.01 \pm 0.00$ & $0.02 \pm 0.00$ & $1.48 \pm 0.11$ \\
As(III) U. lactuca & $0.06 \pm 0.01$ & $0.08 \pm 0.06$ & BDL & $0.01 \pm 0.00$ & $1.55 \pm 0.22$ \\
As(V) artificial diets & $0.05 \pm 0.00$ & $0.07 \pm 0.03$ & $0.01 \pm 0.00$ & $0.01 \pm 0.00$ & $0.99 \pm 0.29$ \\
As(V) G. lemaneiformis & $0.04 \pm 0.02$ & $0.03 \pm 0.01$ & BDL & $0.02 \pm 0.00$ & $1.66 \pm 0.32$ \\
As(V) G.gigas & $0.06 \pm 0.02$ & $0.06 \pm 0.00$ & BDL & $0.02 \pm 0.00$ & $1.61 \pm 0.54$ \\
As(V) U. lactuca & $0.06 \pm 0.01$ & $0.07 \pm 0.00$ & BDL & $0.01 \pm 0.01$ & $2.26 \pm 0.13$ \\
\hline & & & &
\end{tabular}


Table S3 As species concentrations in intestine, liver, and muscle tissues of marine seabass after different dietborne exposure for $28 \mathrm{~d}$. Values are means $\pm \mathrm{SD}(\mathrm{n}=3)$. The foods consist of artificial diets, polychaete, oyster, and clam

\begin{tabular}{|c|c|c|c|c|c|}
\hline & \multicolumn{5}{|c|}{ As species concentrations $(\mu \mathrm{g} / \mathrm{g})$} \\
\hline & As(III) & $\operatorname{As}(V)$ & MMA & DMA & AsB \\
\hline \multicolumn{6}{|c|}{ Intestine } \\
\hline Control & $0.02 \pm 0.00$ & $0.14 \pm 0.01$ & BDL & $0.46 \pm 0.13$ & $2.06 \pm 0.76$ \\
\hline As(III) artificial diets & $0.03 \pm 0.02$ & $0.12 \pm 0.02$ & $0.15 \pm 0.02$ & $0.06 \pm 0.01$ & $3.28 \pm 0.58$ \\
\hline As(III) polychaete & $0.07 \pm 0.01$ & $0.10 \pm 0.04$ & $0.12 \pm 0.03$ & $0.04 \pm 0.01$ & $3.98 \pm 1.32$ \\
\hline As(III) oyster & $0.15 \pm 0.00$ & $0.41 \pm 0.00$ & BDL & $0.41 \pm 0.12$ & $5.01 \pm 1.54$ \\
\hline As(III) clam & $0.09 \pm 0.03$ & $0.10 \pm 0.01$ & $0.14 \pm 0.04$ & $0.88 \pm 0.00$ & $6.14 \pm 0.74$ \\
\hline As( $(V)$ artificial diets & $0.10 \pm 0.03$ & $0.14 \pm 0.01$ & $0.07 \pm 0.04$ & $0.24 \pm 0.03$ & $2.59 \pm 0.32$ \\
\hline $\mathrm{As}(\mathrm{V})$ polychaete & $0.12 \pm 0.06$ & $0.06 \pm 0.01$ & $0.03 \pm 0.02$ & $0.07 \pm 0.01$ & $3.68 \pm 0.90$ \\
\hline $\operatorname{As}(\mathrm{V})$ oyster & $0.09 \pm 0.04$ & $0.10 \pm 0.04$ & $0.05 \pm 0.00$ & $0.48 \pm 0.17$ & $2.43 \pm 0.44$ \\
\hline As(V) clam & $0.15 \pm 0.05$ & $0.22 \pm 0.05$ & BDL & $0.42 \pm 0.07$ & $5.25 \pm 0.02$ \\
\hline \multicolumn{6}{|c|}{ Liver } \\
\hline Control & $0.09 \pm 0.01$ & $0.31 \pm 0.09$ & $0.45 \pm 0.07$ & $0.47 \pm 0.04$ & $0.83 \pm 0.44$ \\
\hline As(III) artificial diets & $0.05 \pm 0.02$ & $0.13 \pm 0.03$ & $0.14 \pm 0.01$ & $0.25 \pm 0.12$ & $1.22 \pm 0.33$ \\
\hline As(III) polychaete & $0.13 \pm 0.01$ & $1.39 \pm 0.66$ & $0.34 \pm 0.03$ & $0.20 \pm 0.09$ & $1.98 \pm 0.07$ \\
\hline As(III) oyster & $0.03 \pm 0.01$ & $0.18 \pm 0.14$ & BDL & $2.89 \pm 1.42$ & $4.97 \pm 0.48$ \\
\hline As(III) clam & $0.02 \pm 0.00$ & $0.17 \pm 0.08$ & BDL & $0.95 \pm 0.19$ & $9.45 \pm 0.64$ \\
\hline $\mathrm{As}(\mathrm{V})$ artificial diets & $0.09 \pm 0.02$ & $0.10 \pm 0.02$ & $0.14 \pm 0.02$ & $0.12 \pm 0.05$ & $2.34 \pm 0.01$ \\
\hline $\mathrm{As}(\mathrm{V})$ polychaete & $0.05 \pm 0.02$ & $0.14 \pm 0.02$ & $0.15 \pm 0.02$ & $0.22 \pm 0.06$ & $2.26 \pm 1.04$ \\
\hline $\mathrm{As}(\mathrm{V})$ oyster & $0.13 \pm 0.04$ & $0.11 \pm 0.02$ & $0.14 \pm 0.04$ & $0.43 \pm 0.20$ & $3.85 \pm 0.39$ \\
\hline $\operatorname{As}(V)$ clam & $0.03 \pm 0.00$ & $0.11 \pm 0.01$ & $0.14 \pm 0.06$ & $1.28 \pm 0.07$ & $9.63 \pm 0.46$ \\
\hline \multicolumn{6}{|c|}{ Muscle } \\
\hline Control & $0.10 \pm 0.06$ & $0.12 \pm 0.07$ & BDL & $0.03 \pm 0.00$ & $4.06 \pm 0.17$ \\
\hline As(III) artificial diets & $0.07 \pm 0.00$ & $0.05 \pm 0.02$ & $0.00 \pm 0.00$ & $0.02 \pm 0.01$ & $3.65 \pm 0.50$ \\
\hline As(III) polychaete & $0.11 \pm 0.01$ & $0.10 \pm 0.03$ & $0.01 \pm 0.00$ & $0.02 \pm 0.01$ & $2.87 \pm 0.13$ \\
\hline As(III) oyster & $0.13 \pm 0.05$ & $0.07 \pm 0.02$ & $0.03 \pm 0.00$ & $0.05 \pm 0.00$ & $4.59 \pm 0.62$ \\
\hline As(III) clam & $0.09 \pm 0.04$ & $0.06 \pm 0.02$ & $0.01 \pm 0.00$ & $0.04 \pm 0.00$ & $5.40 \pm 1.10$ \\
\hline $\operatorname{As}(V)$ artificial diets & $0.10 \pm 0.01$ & $0.10 \pm 0.01$ & $0.02 \pm 0.02$ & $0.04 \pm 0.00$ & $2.94 \pm 0.11$ \\
\hline $\mathrm{As}(\mathrm{V})$ polychaete & $0.09 \pm 0.01$ & $0.08 \pm 0.02$ & $0.03 \pm 0.01$ & $0.02 \pm 0.00$ & $3.48 \pm 0.24$ \\
\hline $\operatorname{As}(\mathrm{V})$ oyster & $0.10 \pm 0.04$ & $0.09 \pm 0.03$ & BDL & $0.02 \pm 0.00$ & $2.57 \pm 0.30$ \\
\hline As $(V)$ clam & $0.13 \pm 0.00$ & $0.08 \pm 0.00$ & BDL & $0.04 \pm 0.01$ & $6.66 \pm 0.01$ \\
\hline
\end{tabular}

